\title{
Radiation Characteristics Enhancement of Dielectric Resonator Antenna Using Solid/Discrete Dielectric Lenses
}

\author{
S.H. Zainud-Deen ${ }^{1}$, W.M. Hassan ${ }^{2 * *}$, Hend A. Malhat ${ }^{1^{*}}$, and K.H. Awadalla ${ }^{1}$ \\ ${ }^{1}$ Faculty of Electronic Eng., Menoufia University \\ ${ }^{2}$ Electronics Research Institute \\ *er_honidal@yahoo.com, **walaaderi.sci.eg
}

\begin{abstract}
The radiation characteristics of the dielectric resonator antennas (DRA) is enhanced using different types of solid and discrete dielectric lenses. One of these approaches is by loading the DRA with planar superstrate, spherical lens, or by discrete lens (transmitarray). The dimensions and dielectric constant of each lens are optimized to maximize the gain of the DRA. A comparison between the radiations characteristics of the DRA loaded with different lenses are introduced. The design of the dielectric transmitarray depends on optimizing the heights of the dielectric material of the unit cell. The optimized transmitarray achieves $7 \mathrm{dBi}$ extra gain over the single DRA with preserving the circular polarization. The proposed antenna is suitable for various applications that need high gain and focused antenna beam.
\end{abstract}

\section{Introduction}

Dielectric resonator antenna (DRA) is an antenna that make use of radiating mode of dielectric resonator (DR). DRA has high radiation efficiency compared with metallic microstrip antennas which having high conductor loss at microwave frequencies. The DRA offers the advantages of small size, different 3-D shapes, a simple structure, low weight, and low cost [1].The point-to-point wireless communication systems require high-gain antennas. Different types of high gain antennas introduced as large fixed-beam antennas, e.g., array antennas [2], reflector antennas [3, 4], reflectarray antennas [5-7], and dielectric lens antennas [8-10]. Modern applications antennas have requirements on size, weight, material cost, packed volume, manufacturing cost, and tolerance to manufacturing errors. Lenses have been widely used in image processing, optical communication, information storage, beam steering, vision correction, 3D displays, and other scientific applications. Lenses can be used to redirect, converge, or diverge electromagnetic radiation. By properly shaping the geometrical configuration and choosing the appropriate material of the lenses, they can transform various forms of divergent energy into a narrow beam. Lens antennas are classified according to the material from which they are constructed, or according to their geometrical shape. Lenses are shaped in order to provide the equal path length condition needed to focus the energy. The closer the focal point, the thicker the lens must be. However, the lens system has some drawbacks: it is inconvenient, inefficient, bulky, and costly. Transmiarrays antennas can be considered as a discrete lens antenna. Transmitarray has the advantages of compact structure, high efficiency, easy operation, and light weight. A discrete lens is an implementation of dielectric material produce enhanced high-directivity reconfigurable beams. Reconfigurable transmitarrays can be used in conformal applications where the physical size and shape of the aperture is constrained $[11,12]$. Half-spherical Luneburg and fisheye lenses, have been realized in the $\mathrm{Ku}$ band as in [13], which realize general 3-D inhomogeneous and nearly isotropic gradientindex material devices. A novel high gain hemispherical lens antenna based on the theory of geometrical optics is presented in [14] for beam scanning application. An extended hemispherical lens illuminated by various printed linear and surface antenna arrays placed at the back of the lens [15]. Large aperture aspheric dielectric lens is proposed for quasi-optics design [16]. Analysis of Hemispherical Dielectric Lens Antennas for Wireless Applications [17].

In this paper, different types of solid dielectric lenses are used to enhance the radiation characteristics of circularlypolarized DRA antenna. The dielectric lenses includes dielectric slab lens, half dielectric sphere lens, complete dielectric sphere lens, and discrete dielectric lens (transmitarray). Each lens type is optimized to enhance the maximum gain of the DRA. The optimization include the lens dimensions material type and spacing from the DRA antenna reflection coefficient, the axial ratio, and the gain of the DRA antenna are investigated. Comparisons between the radiation characteristic of the DRA for different lenses are explained. The full structure of the DRA element with the dielectric lens was analyzed using the finite integration technique (FIT) based simulation CST Microwave Studio software [18]. The results are compared with that calculated by the finite element method (FEM) based simulation technique HFSS software [19]. 


\section{Numerical results}

\subsection{DRA Element loaded with Superstrate Dielectric Slab Lens}

Figure 1 shows the geometry of a circular polarized elliptical DRA element excited using a single feed. The DRA of height $h=3.25 \mathrm{~mm}$, and dielectric permittivity $\varepsilon_{\mathrm{r}}=$ 12 has an elliptical cross section area with aspect ratio $\mathrm{a} / \mathrm{b}=$ 1.5 (major to minor axis ratio), where a semi-major axis $a=$ $4.9 \mathrm{~mm}$. The DRA element is mounted on a square ground plane of side length $20.4 \mathrm{~mm}$. A single feeding probe with length 2.7 and radius $0.25 \mathrm{~mm}$ is embedded with the DRA at a feed point $W_{x}$ and $W_{y}$ of $1.86 \mathrm{~mm}$. The DRA dimensions are optimized to operate at $10 \mathrm{GHz}$ [20]. A planar dielectric slab with dielectric $\varepsilon_{\mathrm{rs}}$ and dimensions of ( $\left.W_{s}, W_{s}, t\right)$ is placed at height $F_{s}$ parallel to the ground plane. The dimensions of the dielectric slab are optimized to maximize the gain of the DRA element at $10 \mathrm{GHz}$. Figure 2 shows the geometry of the DRA loaded with dielectric slab with $\varepsilon_{r s}=12$, length $W_{s}=25.3 \mathrm{~mm}$, and thickness $t=1$ $\mathrm{mm}$, and spacing $F_{s}=20 \mathrm{~mm}$ from DRA. The dielectric slab is operating as a planar lens in front of the DRA antenna.
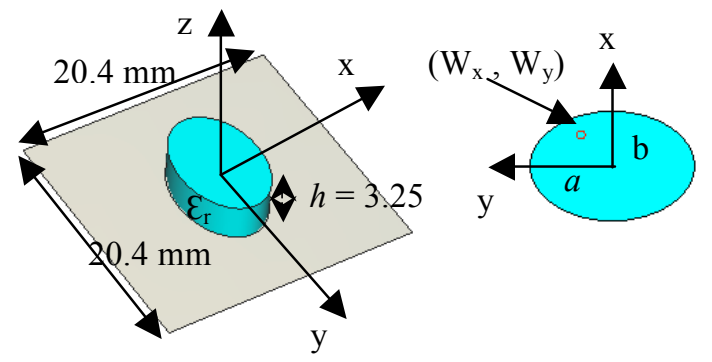

Figure 1: Geometry of a single DRA element on a ground plane.

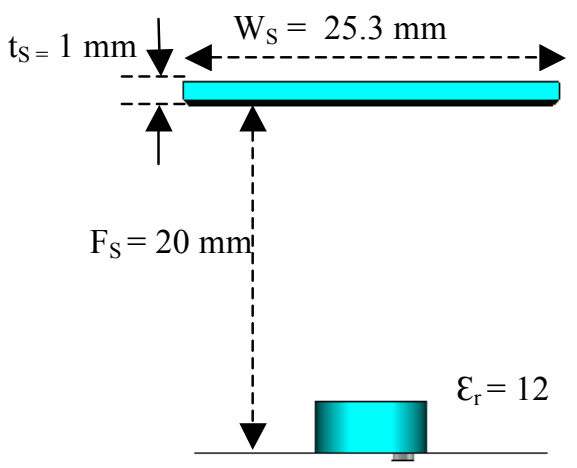

Figure 2: Geometry of a single DRA loaded with a dielectric slab placed at $\mathrm{F}_{\mathrm{S}}$ above the ground plane.

The variation of the reflection coefficients versus frequency for the DRA loaded with the dielectric slab compared to the single DRA as shown in Fig.3. The resonance frequency of the DRA element loaded with the dielectric slab superstrate is $9.7 \mathrm{GHz}$ while for a single DRA is $10 \mathrm{GHz}$. The resonance frequency has gone down due to the effect of the reflections from the slab towards the DRA element and changed of the effective dielectric constant above the DRA. The $-10 \mathrm{~dB}$ impedance bandwidth is increased for the DRA loaded with dielectric slab to about $15.6 \%$. Figure 4 shows the variation of the gain and the axial ratio versus frequency for DRA loaded with dielectric slab and a single DRA. The gain increases to $9.3 \mathrm{~dB}$ for DRA loaded with dielectric slab compared to $6.2 \mathrm{~dB}$ for single DRA at $f=10 \mathrm{GHz}$. The axial ratio is increased to above $5 \mathrm{~dB}$ and the circular polarization is deteriorated. The E- and H-planes of circular polarization radiation patterns for the DRA loaded with the dielectric slab compared to a single DRA at $f=10 \mathrm{GHz}$ are shown in Fig.5. For the DRA loaded with the dielectric slab the R-comp and L-comp represent to the co-polarized and cross-polarized components respectively. The HPBW is $49.6^{\circ}$ in $\mathrm{x}-\mathrm{z}$ plane and $49.3^{\circ}$ in $\mathrm{y}-\mathrm{z}$ plane for the DRA loaded with dielectric slab.

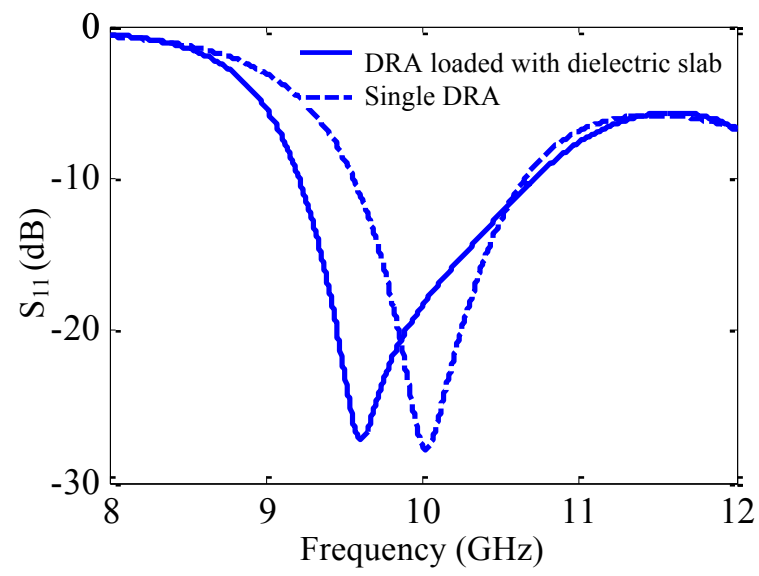

Figure 3: Reflection coefficient versus frequency for DRA loaded with dielectric slab compared to a single DRA.

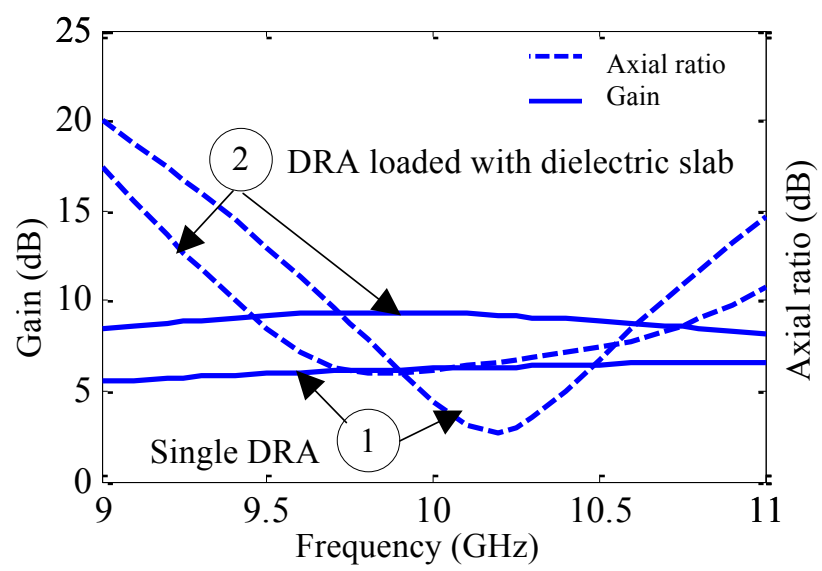

Figure 4: The gain and the axial ratio versus frequency for DRA loaded with dielectric slab compared to a single

\subsection{DRA Element loaded with Lower Half Dielectric Sphere Lens.}

The radiation characteristics of the DRA antenna element can be enhanced by loading a lower half dielectric sphere lens above the DRA element. Figure 6 shows the geometry of a single DRA loaded with lower half dielectric sphere with the diameter $D_{H S}$, the thickness $T_{H S}$, and dielectric constant $\varepsilon_{r l s}$ placed at $F_{H S}$ above the ground plane. 

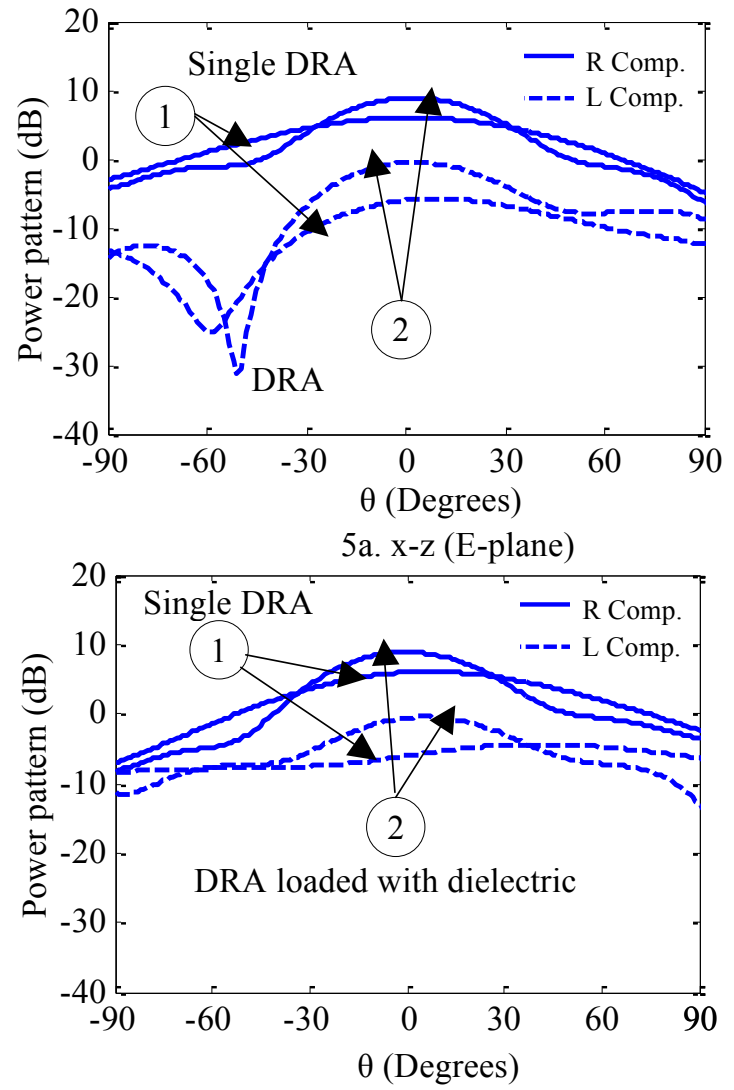

5b. y-z (H-plane)

Figure 5: The E- and H- planes of circular polarization radiation patterns for the DRA loaded with the dielectric slab compared to a single DRA at $f=10 \mathrm{GHz}$

The dimensions of the lower half dielectric sphere dielectric lens are related as follows [2].

$T_{H S}=\frac{1}{n+1}\left[\sqrt{F_{H S}^{2}+\frac{(n+1) D_{H S}}{4(n-1)}}-F_{H S}\right]$,

Where $n=\sqrt{\varepsilon_{r l s}}$.The dimensions of the half sphere lens are optimized to maximize the DRA gain at $10 \mathrm{GHz}$. The half dielectric sphere has $D_{H S}=120 \mathrm{~mm}, F_{H S}=60 \mathrm{~mm}, \mathcal{E}_{r l s}$ $=1.53$, and $T_{H S}=60 \mathrm{~mm}$ are considered. Figure 7 shows the variation of the reflection coefficient versus frequency for DRA loaded with lower half dielectric sphere lens compared to a single DRA. The resonance frequency is shifted down to $9.7 \mathrm{GHz}$ with a reduction in the impedance bandwidth to $9.3 \%$ and a degradation in matching due to the change of the effective dielectric constant above the DRA. The gain and the axial ratio versus frequency for the DRA loaded with lower half dielectric sphere and a single DRA as shown in Fig.8. The DRA gain is increased to 16.1 $\mathrm{dB}$ with circular polarization $3 \mathrm{~dB}$ bandwidth of $250 \mathrm{MHz}$. The E- and H-planes of circular polarization radiation patterns for the DRA loaded with lower half dielectric sphere lens compared to a single DRA at $10 \mathrm{GHz}$ are shown in Fig.9. The HPBW is $14.9^{\circ}$ with cross polarization ratio $10.8 \mathrm{~dB}$.

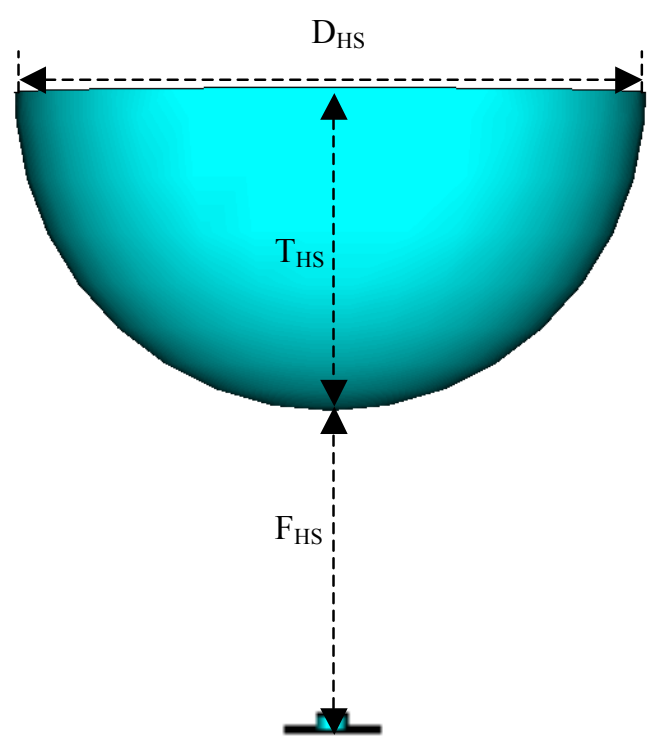

Figure 6: Geometry of a single DRA loaded with lower half dielectric sphere lens.

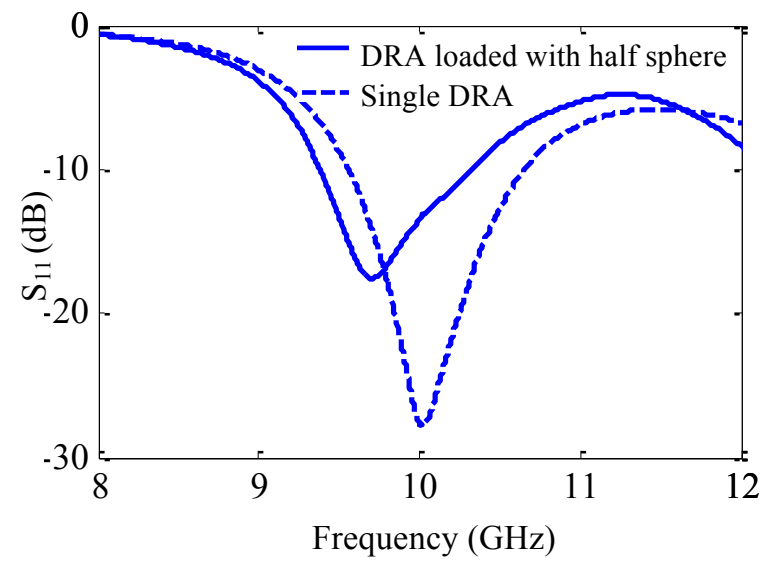

Figure 7: Reflection coefficient versus frequency for DRA loaded with lower half dielectric sphere compared to single DRA.

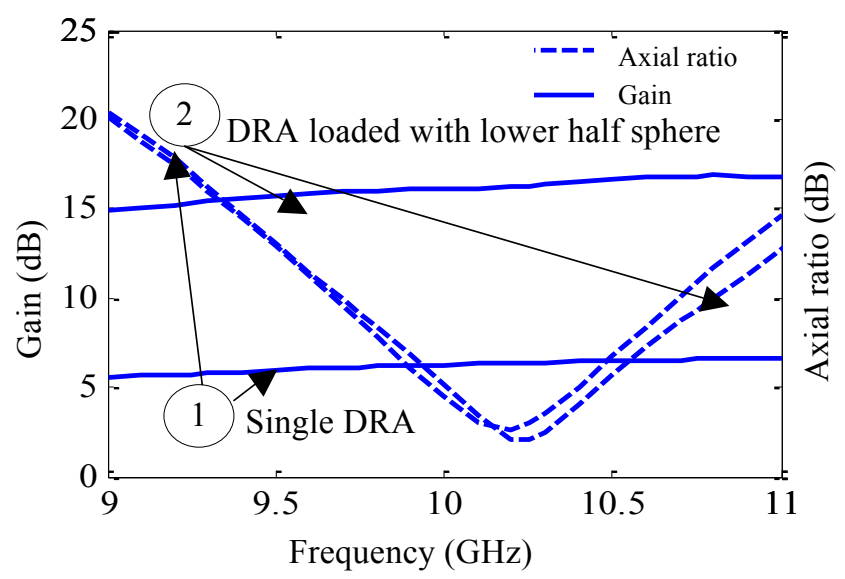

Figure 8: The gain and the axial ratio versus frequency for DRA loaded with lower half dielectric sphere compared to a single DRA. 


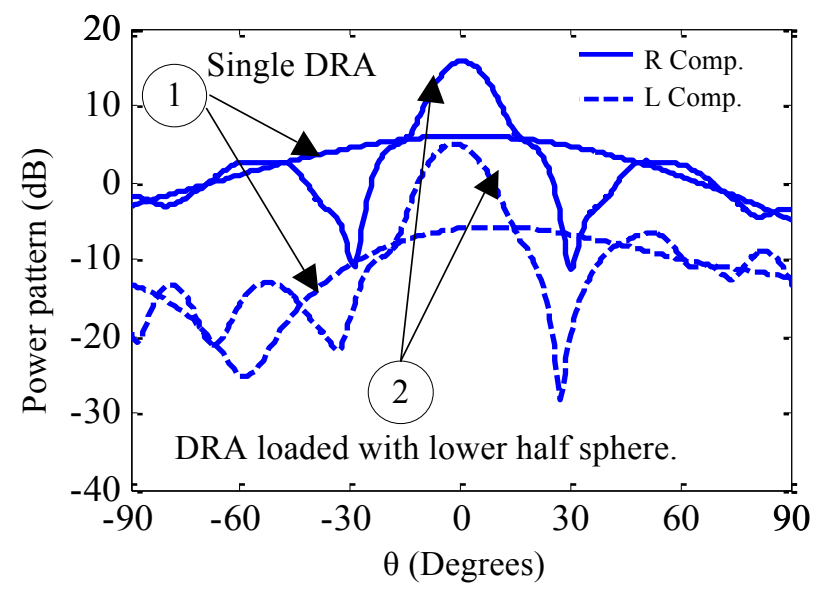

9a. X-z (E-plane)

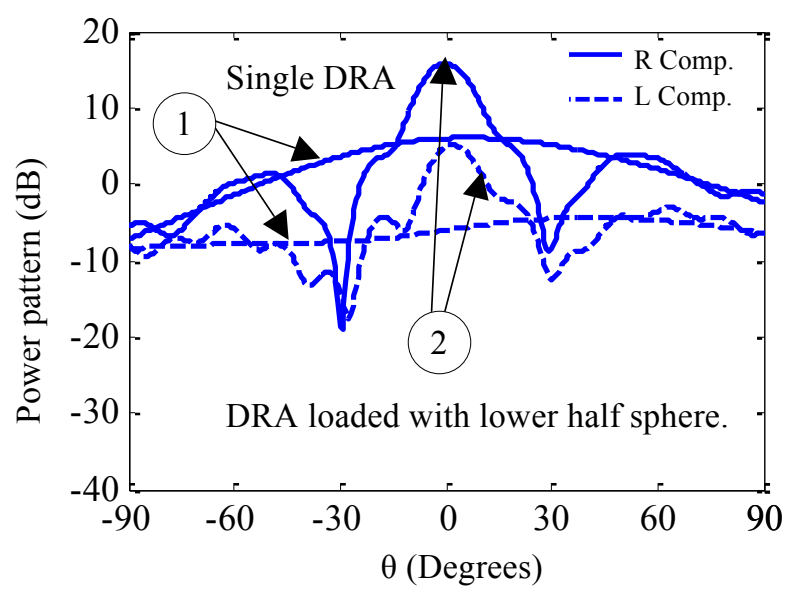

9b. y-z (H-plane)

Figure 9: The E- and H- planes of circular polarization radiation patterns for the DRA loaded with lower half dielectric sphere compared to a single DRA at $f=10$ rUs

\subsection{DRA Element loaded with Upper Half Dielectric Sphere Lens.}

The radiation characteristics of the DRA antenna element can be enhanced by loading upper half dielectric sphere lens above the DRA element. Figure 10 shows the geometry of a single DRA loaded with upper half dielectric sphere with the diameter $D_{H S}$, the thickness $T_{H S}$, and dielectric constant $\varepsilon_{r d s}$ placed at $F_{H S}$ above the ground plane. The dimensions of the upper half sphere dielectric lens are related as follows [2].

$T_{H S}=\frac{\sqrt{4 F_{H S^{2}+D_{H S^{2}}}-2 F_{H S}}}{2(n-1)}$,

The dimensions of the upper half dielectric sphere lens are optimized to maximize the DRA gain at $10 \mathrm{GHz}$. The thickness $T_{H S}=60 \mathrm{~mm}$, the diameter $D_{H S}=120 \mathrm{~mm}$, the distance $F_{H S}=60 \mathrm{~mm}$, and the dielectric constant $\varepsilon_{r u s}=2$. Figure 11 shows the variation of the reflection coefficient versus frequency for DRA loaded with upper half dielectric sphere lens compared to a single DRA. The resonance frequency is shifted down to $9.69 \mathrm{GHz}$ with a reduction in the impedance bandwidth to $10.3 \%$ and a degradation in matching due to the change of the effective dielectric constant above the DRA. The gain and the axial ratio versus frequency for the DRA loaded with upper half sphere and a single DRA as shown in Fig.12. The DRA gain is increased to $19 \mathrm{~dB}$ with circular polarization $3 \mathrm{~dB}$ bandwidth of 270 $\mathrm{MHz}$. The E- and H-planes of circular polarization radiation patterns for the DRA loaded with upper half dielectric sphere lens at $10 \mathrm{GHz}$ are shown in Fig.13. The HPBW is $13.4^{\circ}$ with cross polarization ratio $12 \mathrm{~dB}$. It can be observed that the difference between the lower half sphere and the upper half sphere is quite small, apart from the higher gain obtained by the upper half sphere. This can be attributed to the fact that, when both half spheres have their lowest point at the same distance from the center of the DRA, the power intercepted by the upper half sphere is more than the power intercepted by the lower one.

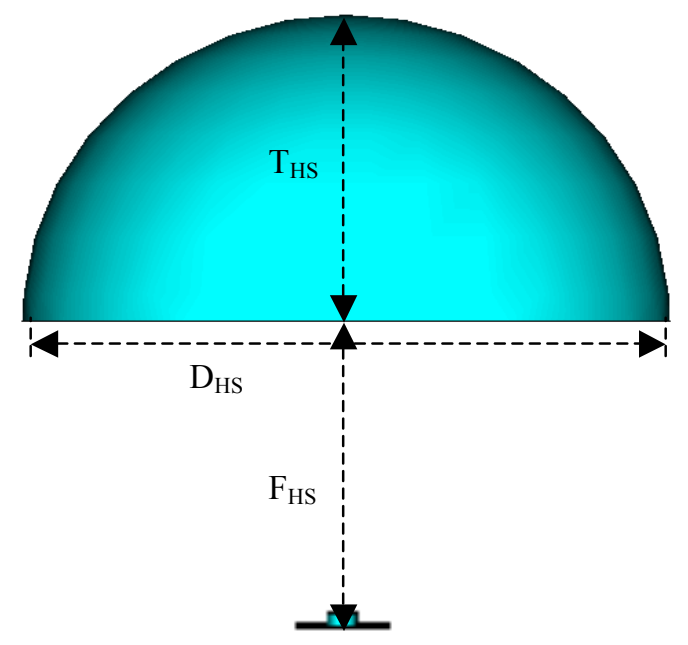

Figure 10: Geometry of a single DRA loaded with upper half dielectric sphere.

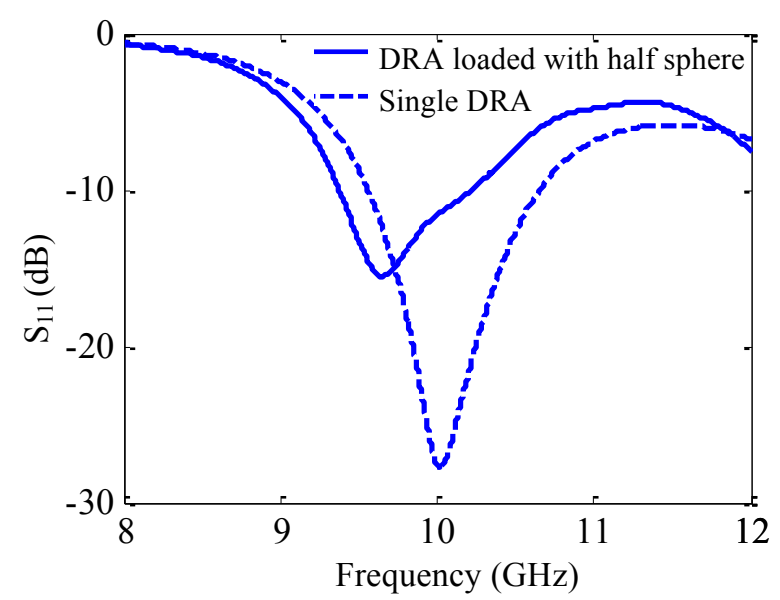

Figure 11: Reflection coefficient versus frequency for DRA loaded with upper half dielectric sphere compared to single DRA. 


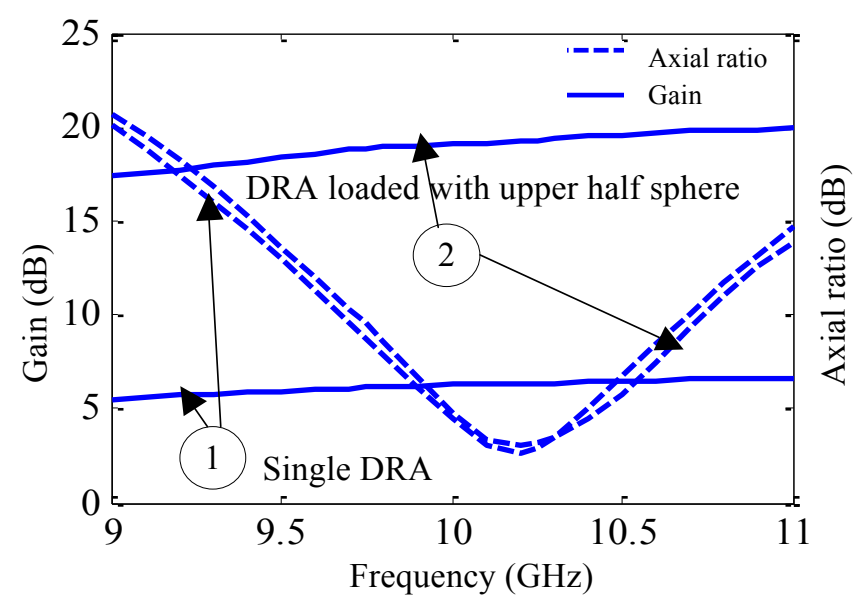

Figure 12: The gain and the axial ratio versus frequency for DRA loaded with upper half dielectric sphere compared to a single DRA.

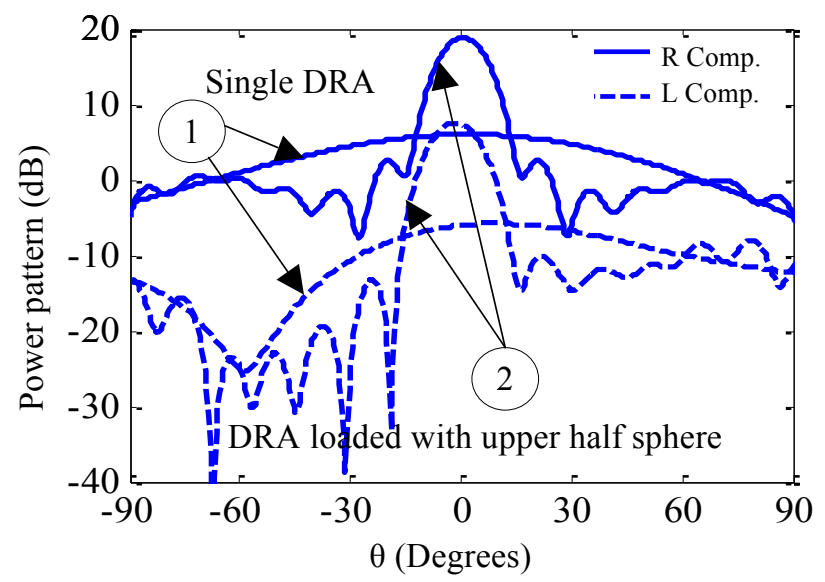

13a. x-z (E-plane)

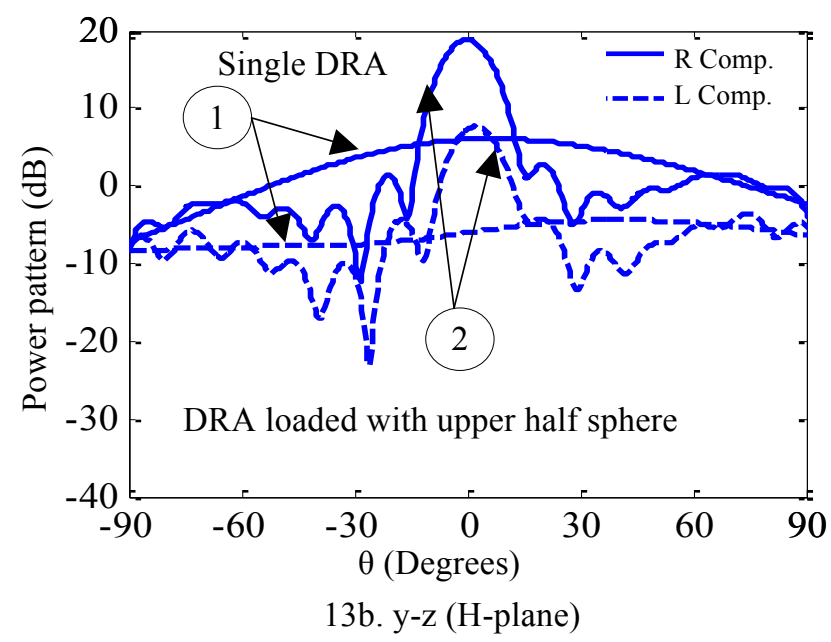

Figure 13: The E- and H- planes of circular polarization radiation patterns for the DRA loaded with upper half dielectric sphere compared to a single DRA at $f=10 \mathrm{GHz}$.
2.4. DRA Element loaded with Dielectric Sphere Lens.

The radiation characteristics of the DRA antenna element can be enhanced by loading a dielectric sphere lens above the DRA element. Figure 14 shows the geometry of a single DRA loaded with a dielectric sphere with the diameter $D_{S}$, the thickness $T_{S}$, and dielectric constant $\varepsilon_{r d s}$ placed at $F_{S}$ above the ground plane. All the above dimensions are related to [2].

$T_{5}=\sqrt{\frac{4(n-1) F_{5}^{2}-(n-3) D_{5}^{2}}{4(n-1)(n-3)^{2}}}+\frac{F_{5}}{\mathrm{n}-3}$

The dimensions of the dielectric sphere lens are optimized to maximize the DRA gain at $10 \mathrm{GHz}$. The dielectric sphere dimensions has thickness $T_{S}=120 \mathrm{~mm}$, the diameter $D_{S}=$ $120 \mathrm{~mm}$, the distance $F_{S}=60 \mathrm{~mm}$, and the dielectric constant $\varepsilon_{r d s}=1.53$. Figure15 shows the variation of the reflection coefficient versus frequency for DRA loaded dielectric sphere lens compared to a single DRA. The resonance frequency is shifted down to $9.7 \mathrm{GHz}$ with reduction in impedance bandwidth to $9.3 \%$ and degradation in matching. The gain and the axial ratio versus frequency for the DRA loaded with dielectric sphere and a single DRA. The DRA gain is enhanced to $15.2 \mathrm{~dB}$ with circular polarization $3 \mathrm{~dB}$ bandwidth of $200 \mathrm{MHz}$ as shown in Fig.16. Finally, Fig.17 shows the simulated circularly polarized components in the E- and H-planes for the DRA loaded with dielectric sphere lens at $f=10 \mathrm{GHz}$. The $\mathrm{HPBW}$ is $14.8^{\circ}$ with cross polarization ratio $11.3 \mathrm{~dB}$. It has to be noted here that the gain of the complete sphere lens is lower than the upper half sphere lens when the height of the lowest point of the lens above the ground plane in both cases is the same. This, again, is attributed to the larger intercepting area for the radiated energy from the DRA in the case of the upper half sphere lens, which intercepts larger amount of radiated energy relative to the complete sphere case and the lower half sphere case. The intercepted amount of energy for the lower half sphere lens is the same as that for the complete sphere lens, therefore, the gain in these two cases is almost the same.

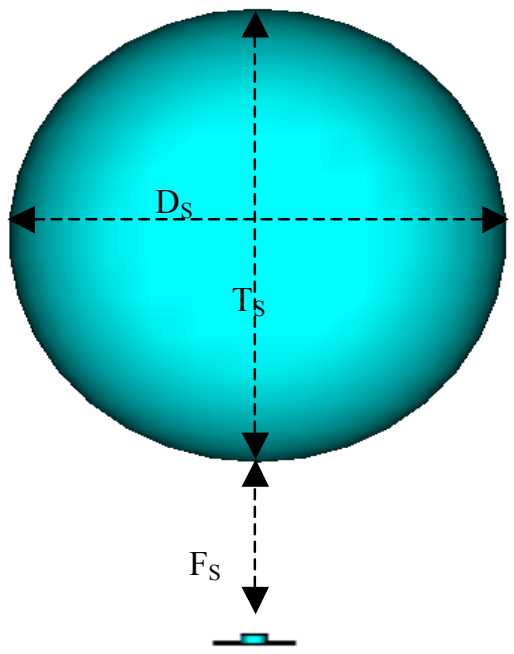

Figure 14: Geometry of a single DRA loaded with upper dielectric sphere. 


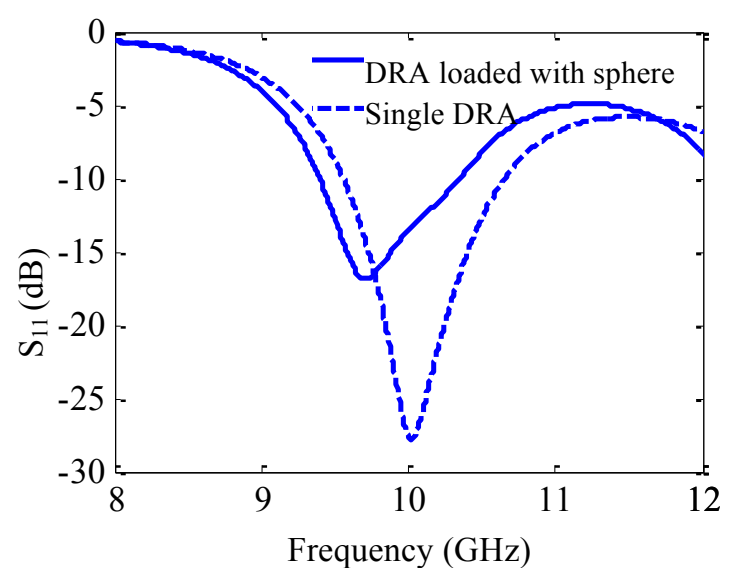

Figure 15: Reflection coefficient versus frequency for DRA loaded with dielectric sphere compared to single DRA.

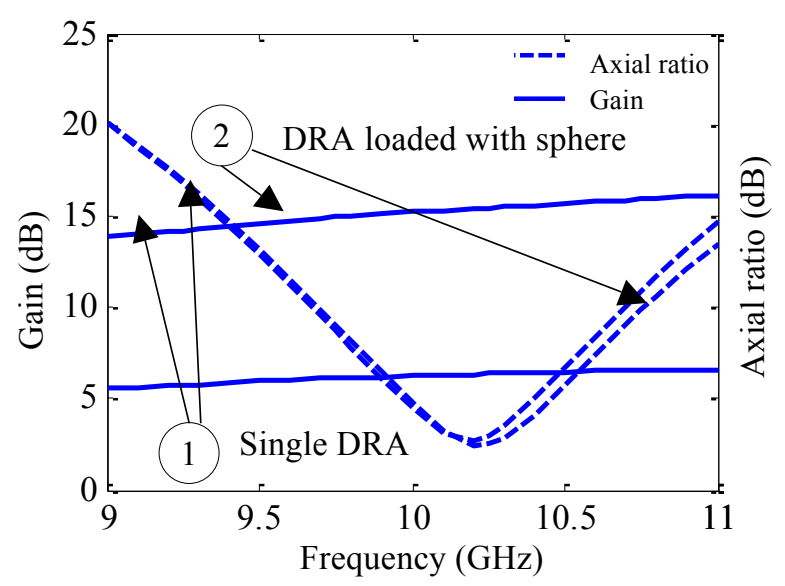

Figure 16: The gain and the axial ratio versus frequency for DRA loaded with dielectric sphere compared to a single DRA.

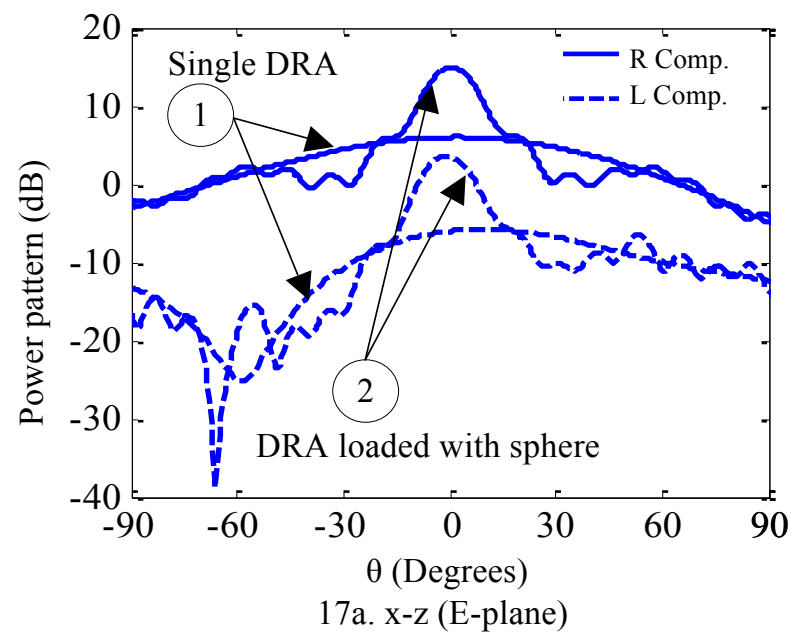

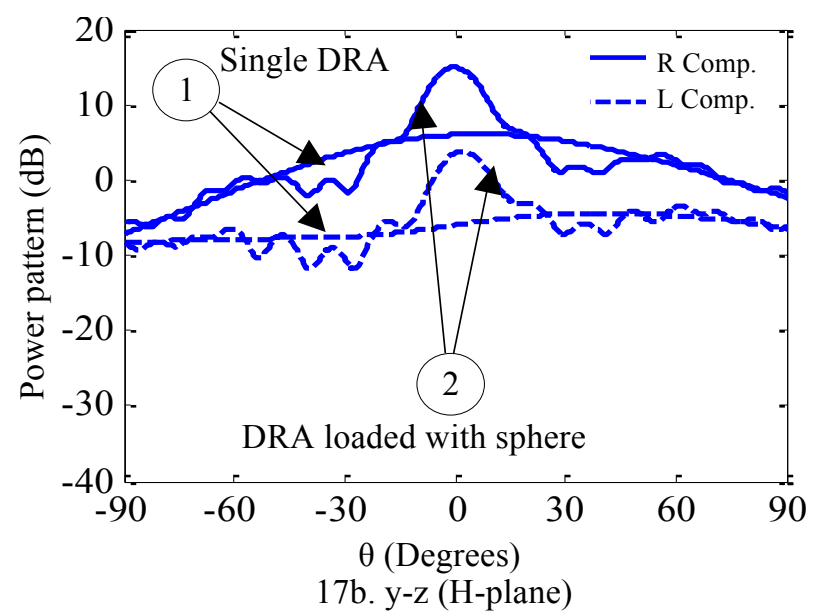

Figure 17: The E- and H- planes of circular polarization radiation patterns for the DRA loaded with dielectric sphere compared to a single DRA at $f=10 \mathrm{GHz}$.

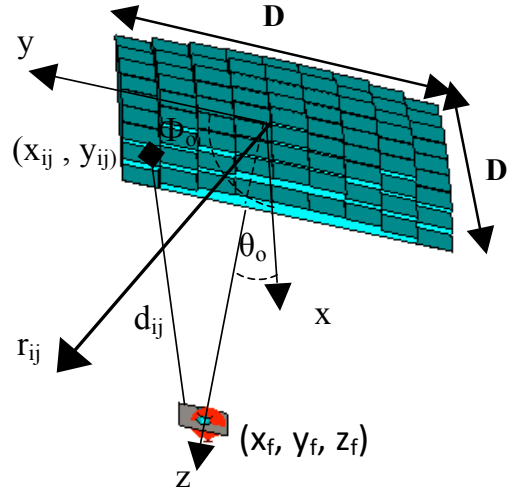

3-D view

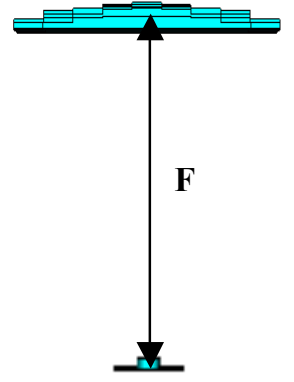

Side view Figure 18: Geometry of the DRA loaded with transmitarray.

\subsection{DRA Element with Discrete Dielectric Planar Lens.}

Transmitarrays are available architectures for achieving high-directivity beam for beam forming applications [12]. The two most important design criteria for a transmitarray element are its phase range and insertion loss. Firstly, in order to design an element suitable for an arbitrarily large array where there is possibly phase wrapping in the aperture fields, a phase tuning range of $360^{\circ}$ is required. Secondly, the insertion loss must be minimized. The DRA is an attractive element for transmitarrays as it is an excellent radiator, and has a negligible metallic loss [21, 22]. In addition to other advantages, such as small size, high radiation efficiency, low weight, low cost and wide bandwidth. Also, it can be used when operating at millimeter and microwave frequencies. The required phase compensation distribution $\varphi_{i j}$ at each unit cell element in the transmitarray to collimate a beam in the $\left(\theta_{0}, \emptyset_{0}\right)$ direction is obtained by [23]:

$\varphi_{i j}\left(x_{i j}, y_{i j}\right)=k_{o}\left[\begin{array}{c}d_{i j}-x_{i j} \sin \left(\theta_{0}\right) \cos \left(\emptyset_{0}\right)- \\ y_{i j} \sin \left(\theta_{0}\right) \sin \left(\emptyset_{0}\right)\end{array}\right]$, 
$d_{i j}=\sqrt{\left(x_{i j}-x_{f}\right)^{2}-\left(y_{i j}-y_{f}\right)^{2}-z_{f}^{2}}$,

where $k_{o}$ is the propagation constant in vacuum, $d_{i j}$ is the distance from the feed point $\left(x_{f}, y_{f}, z_{f}\right)$ to the position of the element $i j^{\text {th }}$ in the array and $\left(x_{i j}, y_{i j}\right)$ are the coordinates of the cell element as shown in Fig.18. The phase of the transmitted wave of each element is achieved by varying the element dimensions. Transmitarrays are constructed of 2-D arrangement of unit cells. The configuration of the proposed unit cell is shown in Fig.19a. To calculate the required phase compensation for each unit cell, the cell is placed inside a waveguide simulator as shown in Fig.19b. The perfect electric and magnetic wall boundary conditions are applied to the sides of the surrounding waveguide, and result in image planes on all sides of the unit cell to represent an infinite array approximation. A plane wave was used as the excitation of the unit cells inside the waveguide simulator and only normal incidence angle was considered. There are several limitations to the infinite array approach. First, all elements of the transmitarray are identical; this is clearly not the case in the real transmitarray in which the height of the dielectric box in each cell element must vary according to the required phase compensation. Secondly, the transmitarray itself is not infinite in extent.

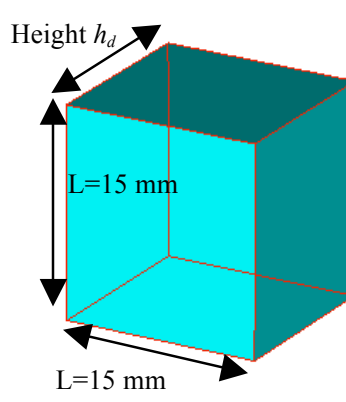

19 a: Unit cell

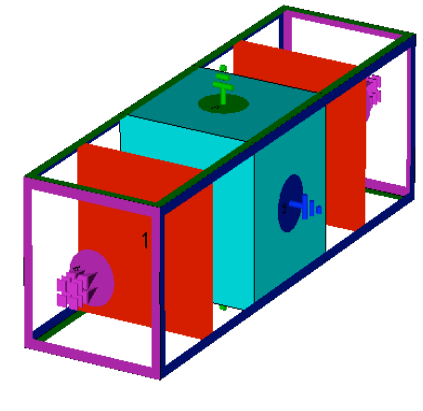

19 b: waveguide simulation
Figure 19: The detailed dimension of transmitarray unit cell and waveguide simulation.

The cell element of the transmitarray consists of square dielectric cell with arm length $15 \mathrm{~mm}$, height $h_{d}$ and a relative permittivity of $\mathcal{E}_{r}=12$. The variation of the transmission magnitude and phase versus the height of the unit cell as shown in Fig. 20. The relationship between variable height and the transmission coefficient at $10 \mathrm{GHz}$ was determined using the FIT and FEM techniques. A good agreement between the two techniques. The height of the unit cell varied from $0.1 \mathrm{~mm}$ to $12 \mathrm{~mm}$ produce a phase shift ranging from $0^{\circ}$ to $350^{\circ}$ with the transmission coefficient magnitude changes from $0 \mathrm{~dB}$ to $-5.4 \mathrm{~dB}$. The variation of the phase shift with the height is straight forward and, as expected, it is almost linear. However, the variation of the transmission coefficient magnitude with the height is varying within about $-5.4 \mathrm{~dB}$ and is periodic in nature. This variation characteristics is attributed to the internal reflections of the wave between the upper and lower faces of the dielectric cell. The internal reflections are adding in phase to the forward radiation at certain heights and adding in anti-phase for the middle heights in between the positive peaks.

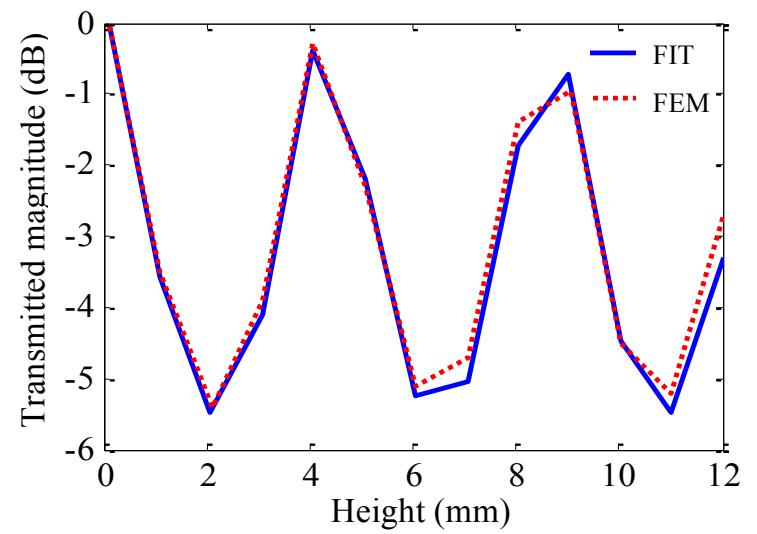

(a)

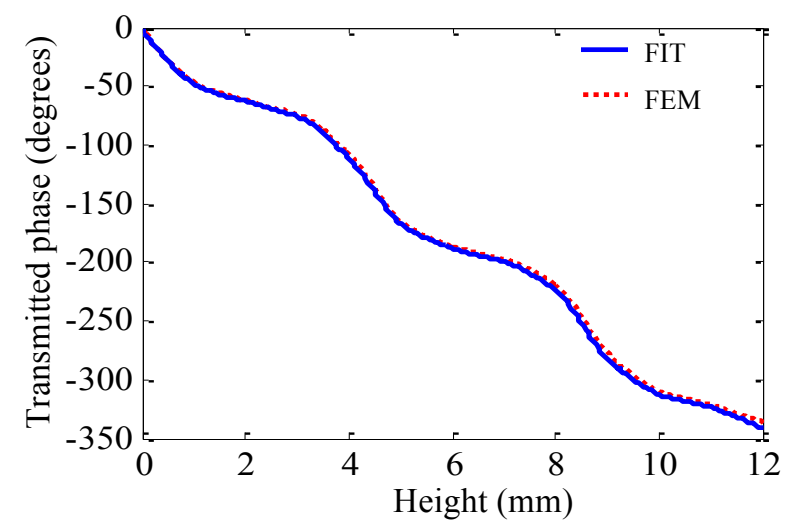

(b)

Figure 20: The variation of transmission magnitude (a) and nhase $(\mathrm{h})$ versus height.

The DRA is loaded with $9 \times 9$ unit cell transmitarray design at $10 \mathrm{GHz}$ and covering an area of $135 \times 135 \mathrm{~mm}^{2}$. The transmitarray is constructed from one piece of etched dielectric material sheet. The simplicity of the structure makes it practical in terms of cost, space, and ease of fabrication. The transmitarray distance from the DRA $F / D$ is optimized for lower side lobe levels and highest DRA gain. The radiation characteristic of the DRA loading with transmitarray with different heights F/D are listed in Table 1.The maximum DRA gain of $17.9 \mathrm{~dB}$ is achieved for the transmitarray at $F / D=0.9$. The simulated results for the gain and axial ratio of the DRA element loaded with the optimized transmitarray versus frequency are calculated and compared with single DRA element as shown in Fig.21. The gain from the transmitarray is achieved which reached to $17.9 \mathrm{~dB}$. 1-dB gain bandwidth of $2.5 \mathrm{GHz}$ is achieved, the circular polarization radiation characteristics are maintained with 3-dB axial ratio $\mathrm{BW}=200 \mathrm{MHz}$. The Eand $\mathrm{H}$-planes of circular polarization radiation patterns for the DRA loaded with transmitarray lens at $10 \mathrm{GHz}$ are 
shown in Fig.22. The HPBW is $11.4^{\circ}$ with cross polarization ratio $11.2 \mathrm{~dB}$. The transmitarray arrangement is more directive, light weight when optimized in the design compared to the superstrate and the dielectric lens structures. Table 2 introduces a comparison between the radiation characteristic of the DRA element loaded with different types of lenses. From the comparison, it seems that the loading of the DRA with upper half sphere gives the maximum gain but the structure is bulky. However, loading the DRA with transmitarray introduces high gain with simple structure and can be increased by increasing the transmitarray size.

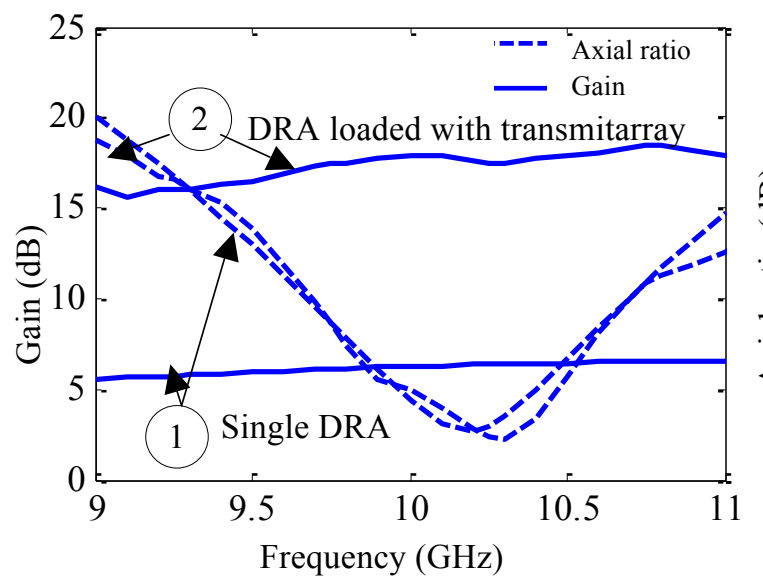

Figure 21: The gain and the axial ratio versus frequency for DRA loaded with transmitarray compared to a single DRA.

Table 1: The radiation characteristics of $9 \mathrm{x} 9$ etching transmitarray fed by single elliptic DRA element for different ratios F/D.

\begin{tabular}{ccc}
\hline $\begin{array}{c}\text { Array position } \\
\text { F/D }\end{array}$ & $\begin{array}{c}\text { Gain in } \mathrm{dB} \\
\text { at } f=10 \mathrm{GHz}\end{array}$ & $\begin{array}{c}\text { Center frequency } \\
\text { B.W }\end{array}$ \\
\hline 1 & 14.5 & $9.3 \%$ \\
0.9 & 17.9 & $8.5 \%$ \\
0.8 & 15.6 & $7.8 \%$ \\
0.7 & 17.3 & $8.5 \%$ \\
0.6 & 16.7 & $7.6 \%$ \\
0.5 & 17.5 & $8.8 \%$ \\
\hline
\end{tabular}
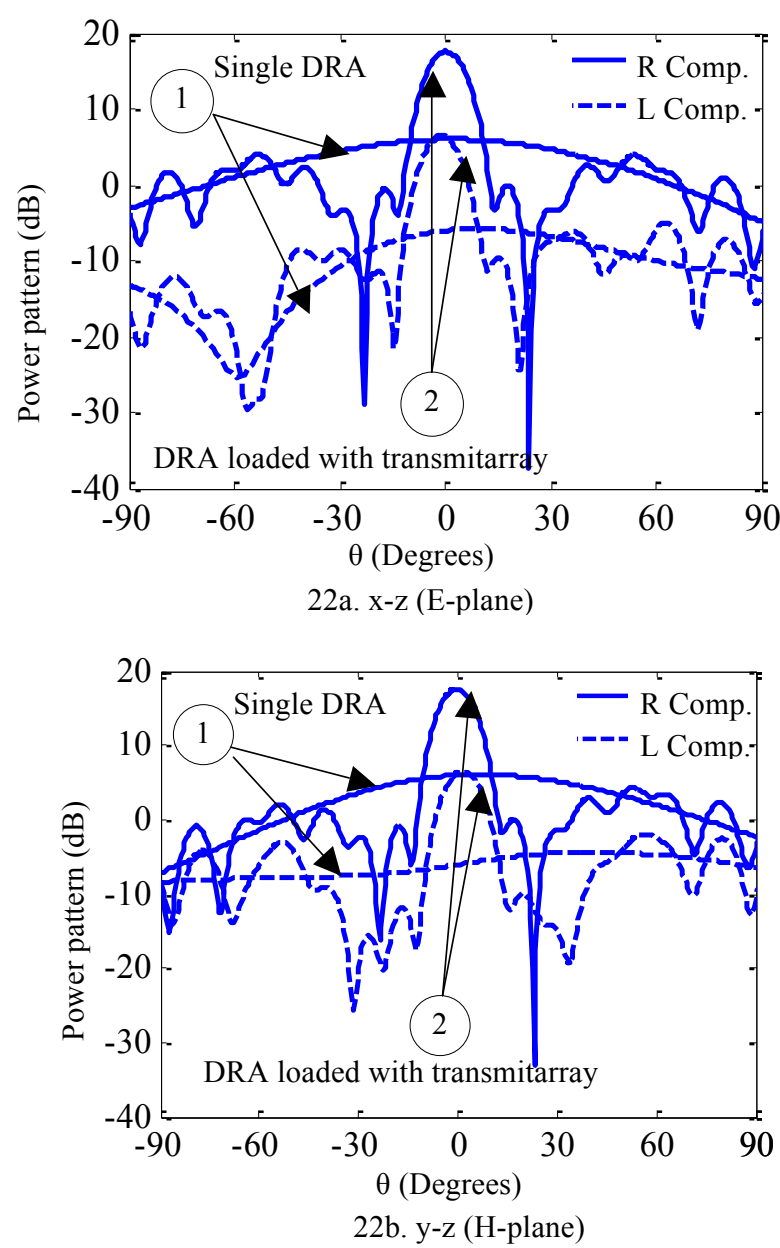

Figure 22: The E- and H- planes of circular polarization radiation patterns for the DRA loaded with transmitarray compared to a single DRA at $f=10 \mathrm{GHz}$.

Table 2: Comparison between different enhanced radiation characteristic for the DRA element loaded with different types of lenses

\begin{tabular}{|c|c|c|c|c|c|c|}
\hline & $\begin{array}{c}\text { Single } \\
\text { DRA }\end{array}$ & $\begin{array}{l}\text { Dielectric } \\
\text { slab }\end{array}$ & $\begin{array}{c}\text { Lower half } \\
\text { dielectric sphere }\end{array}$ & $\begin{array}{c}\text { Upper half } \\
\text { dielectric sphere }\end{array}$ & $\begin{array}{l}\text { Dielectric } \\
\text { sphere }\end{array}$ & $\begin{array}{c}\text { Dielectric } \\
\text { transmitarray }\end{array}$ \\
\hline Impedance B.W \% & $11 \%$ & $15.6 \%$ & $9.3 \%$ & $10.3 \%$ & $9.3 \%$ & $10.2 \%$ \\
\hline Gain $(\mathrm{dB})$ & 6.3 & 9.3 & 16.1 & 19 & 15.2 & 17.9 \\
\hline Angular width (3dB) & $87.1^{\circ}$ & $49.3^{\circ}$ & $14.9^{\circ}$ & $13.4^{\circ}$ & $14.8^{\circ}$ & $11.4^{\circ}$ \\
\hline$E_{L} / E_{R}(d B)$ & 12.2 & 9.4 & 10.8 & 12 & 11.3 & 11.2 \\
\hline $1 \mathrm{~dB}$ gain $\mathrm{B} . \mathrm{W}(\mathrm{GHz})$ & 3.9 & 1.9 & 3.9 & 3.8 & 3.9 & 2.5 \\
\hline Lens Size & - & $0.64 \mathrm{~cm}^{3}$ & $452.4 \mathrm{~cm}^{3}$ & $452.4 \mathrm{~cm}^{3}$ & $904.8 \mathrm{~cm}^{3}$ & $111.825 \mathrm{~cm}^{3}$ \\
\hline $1^{\text {st }}$ side lobe level & - & $4 \mathrm{~dB}$ & $14 \mathrm{~dB}$ & $16 \mathrm{~dB}$ & $9.2 \mathrm{~dB}$ & $17.9 \mathrm{~dB}$ \\
\hline Height & - & $21 \mathrm{~mm}$ & $120 \mathrm{~mm}$ & $120 \mathrm{~mm}$ & $180 \mathrm{~mm}$ & $125.52 \mathrm{~mm}$ \\
\hline
\end{tabular}




\section{Conclusion}

In paper, the radiation characteristics enhancement of the DRA element loaded with solid/discrete dielectric lenses are investigated. Different types of solid dielectric lenses have been investigated. Each lens is optimized to maximize the radiation characteristics of the DRA. The DRA loaded with a planar dielectric sheet introduces maximum of $9.3 \mathrm{~dB}$ with widest BW of $15.6 \%$. The DRA is loaded with different arrangement of dielectric lens sphere. Dielectric transmitarray constructed from one piece of etched dielectric material sheet is loaded to the DRA. The dielectric sheet is composed of 9x9 unit cell elements and has covered an area of $13.5 \times 13.5 \mathrm{~cm}^{2}$. The height of each cell element is optimized to maximize the transmission coefficient through the structure at $f=10 \mathrm{GHz}$. The full wave analysis is used to explain the impedance bandwidth, the gain, the angular width, and the $1 \mathrm{~dB}$ gain bandwidth for each construction. The gain from the transmitarray is increased to $17.9 \mathrm{~dB}$. The transmitarray arrangement is more directive relative to the superstrate, the lower half sphere lens, and the full sphere lens with angular width $(3 \mathrm{~dB}) 11.4^{\circ}$. Cross-polarized $\mathrm{E}_{\mathrm{L}} / \mathrm{R}_{\mathrm{R}}$ ratio is $11.4 \mathrm{~dB}$. The $1^{\text {st }}$ side lobe relative to the main beam is $17.9 \mathrm{~dB}$. The $\mathrm{B} . \mathrm{W}$ is $1 \mathrm{GHz}$ and light weight when optimized in the design compared to the superstrate and the dielectric lens structures. In addition to getting more design flexibility using the given transmitarray as it can be tailored to any desired application.

\section{References}

[1] R.K. Mongia and P. Bhartia, "Dielectric Resonator Antennas: AReview and General Design Relations for Resonant Frequency and Bandwidth," Int. J. Microwave Millimeter-Wave Eng., vol. 4, no. 3, pp. 230-24, July 1994.

[2] Y. T. Lo, and S. W. Lee, Antenna Handbook: Theory, Applications, and Design, New York, USA, Springer; 1 edition, June 30, 1988.

[3] C.A. Balanis, Antenna Theory, Analysis and Design, $3^{\text {rd }}$ Ed, New York, USA, John Wiley and Sons, 2005.

[4] M. Ruphuy, Z. Ren, and O.M. Ramahi, "Flat Far Field lenses and reflectors," Progress In Electromagnetics Research M, (PIER M), vol.34, pp.163-170, 2014.

[5] J. Huang and J.A. Encinar, Reflectarray Antennas, IEEE Press, New Jersey, USA, John Wiley and Sons, 2008.

[6] M.K. Al-Nuaimi and W. Hong, "Discrete Dielectric Reflectarray and Lens for E-Band with Different Feed," IEEE Antennas and Wireless Propagat. Letters, vol.13, pp.947-950, 2014.

[7] S.H. Zainud-Deen, N. El-Shalaby, S.M. Gaber, H.A. Malhat, and K.H. Awadalla, "Reflectarrays Mounted on or Embedded in Cylindrical or Spherical surfaces," 2012 Middel East Conference on Antennas and Propagat. (MECAP 2012), Cairo, Egypt, pp.1-6, 2012.

[8] L. Dussopt, H. Kaouach, J. Lanteri, and R. Sauleau, "Circularly-Polarized Discrete Lens Antennas in $60-\mathrm{GHz}$ Band," Radio engineering, vol.20, no.4, pp.733-738, Dec. 2011.

[9] S.H. Zainud-Deen, S.M. Gaber, H.A. Malhat, and K.H. Awadalla, " Perforated Transmitaray-Enhanced Circularly Polarized Antenna for High-Gain Multi-Beam Radiation,"
2013 International Symposium on Antennas and Propagation (ISAP 2013), Nanjing, China, Oct.2013.

[10] J. S. Chieh, C. Meagher, and D. Hooper,"A L-Band Superstrate Lens Enhanced Antenna and Array for Tactical Operations," IEEE Int. Symp. On Antennas and Propagation and USNC-URSI Nat. Radio Science Meeting, Orlando, FL, USA, July 2013.

[11] J. Y. Lau and S. V. Hum, "A Wideband Reconfigurable Transmitarray Element," IEEE Trans. Antennas Propagat., vol. 60, no. 3, pp. 1303-1311, March 2012.

[12] J. Y. Lau and S. V. Hum, "Reconfigurable Transmitarray Design Approaches for Beamforming Applications," IEEE Trans. Antennas Propagat., vol. 60, no. 12, pp. 5679-5689, Dec. 2012.

[13] P. Piksa, P. Cernynear,"Near-Field Measurement of Gaussian Beam behind Dielectric Lens" Radioelektronic, 17th International Conference, pp.1-4, April 2007.

[14] S.Ravishankar, T.S.Rukmini, H.VKumaraswamy, S.Sunit, Y.Karthik ,P.Vinay and Arjun Ravishankar "Analysis of Hemispherical Dielectric Lens Antennas for WirelessApplications" Electromagnetics,APACE,pp. 1-7, DEC 2007

[15] J. P. Thakur, W.-G. Kim, and Y.-H. Kim "Large Aperture Low Aberration Aspheric Dielectric Lens Antenna for WBand Quasioptics" Progress In Electromagnetics Research, PIER 103, pp. 57-65, 2010

[16] N. T. Nguyen, R. Sauleau, M. Ettorre, and L. Le Coq "Focal Array Fed Dielectric Lenses: An Attractive Solution for Beam Reconfiguration at Millimeter Waves" IEEE Transactions On Antennas And Propagation, VOL. 59, NO. 6, JUNE 2011

[17] J. Qiu, W. Li, and Y. Suo "A Novel High Gain Beam Scanning Hemispherical Dielectric Lens Antenna"6th International Conference on ITS Telecommunications Proceedings, 2006.

[18] O.C. Zienkiewicz, R.L. Taylor, and J.Z. Zhu, The Finite Element Method: Its Basis and Fundamentals, $6^{\text {th }} \mathrm{Ed}$, Elsevier, Butterworth-Heinemann, UK, 2005.

[19] R. Marklein, "The Finite Integration Technique As A General Tool to Compute Acoustic, Electromagnetic, Elastodynamic and Coupled Wave Fields," Review of Radio Science, Piscataway, USA, IEEE Press, 2002.

[20] Hend A. Malhat, S.H. Zainud-Deen, S.M. Gaber, and K. H. Awadalla, " Multilayer dielectric resonator antenna transmitarray for near-field and far-field fixed RFID reader," Progress In Electromagnetics Research C, PIER C, Vol. 27, pp. 129-142, 2012.

[21] A. Petosa and A. Ittipiboon, "Dielectric Resonator Antennas: A Historical Review and the Current State of the Art," IEEE Antennas Propagat. Mag., vol.52, no.5, pp.91-116, Oct. 2010.

[22] A. A. Kishk, "DRA-Array with 75\% Reduction in Elements Number," Radio and Wireless Symposium (RWS), Austin, TX, USA, pp. 70-72, Jan. 2013.

[23]S. M. Gaber, Analysis and design of reflectarrays/transmitarrays antennas, Ph.D. Thesis, Minoufiya University, 2013. 